Muro de la investigación, 2018: 3(2), agosto-diciembre,

ISSN: $2523-2886$

DOI: https://doi.org/10.17162/rmi.v3i2.1158

\title{
Ciclo pastoral, "síndrome de burnout" y satisfacción laboral en pastores de la Unión Central Brasileira, São Paulo, 2017
}

\author{
Gérson Jorge Quiles ${ }^{1}$ y Daniel William Richard Pérez ${ }^{1 \mathrm{a}}$ \\ Universidad Peruana Unión, Perú ${ }^{1}$
}

Recibido: 12 abril de 2018

Aceptado: 15 de junio de 2018

\section{Resumen}

El llamado "ciclo pastoral" (CP) es uno de los problemas más resaltantes en la obra eclesial en varias latitudes del mundo cristiano-evangélico. Inclusive en la misma Iglesia Adventista del Séptimo Día (IASD), este problema afecta al crecimiento de la iglesia y al entorno familiar del pastor. No solo ello, algunas de estas implicaciones resultan en casos del llamado "síndrome de burnout", que puede tener relación con la duración del CP. Es por ello que la presente investigación se refiere a la relación entre el ciclo pastoral con el síndrome de burnout y la consecuencia que esto conlleva en la satisfacción laboral en 395 pastores distritales de la Unión Central Brasileira (UCB), São Paulo, 2017. El presente trabajo de investigación es de enfoque cuantitativo, y usa un diseño tanto transversal como correlacional. La técnica de recolección de datos fue por medio de la encuesta, la que fue dirigida a pastores de la UCB.

Palabras clave: Ciclo pastoral; síndrome de Burnout; satisfacción laboral; pastor

\section{Pastoral cycle, "burnout syndrome" and job satisfaction in pastors of the Brazilian Central Union, São Paulo, 2017}

\begin{abstract}
The so-called "pastoral cycle" $(\mathrm{CP})$ is one of the most prominent problems in the ecclesial work in various latitudes of the Christian-evangelical world. Even in the same Seventh-day Adventist Church (SDA, IASD in spanish), this problem affects the growth of the church and the pastor's family environment. Not only this, some of these implications lost in cases of the so-called "burnout syndrome", which may be related to the duration of CP. That is why this research refers to the relationship between the pastoral cycle with burnout syndrome and the consequence that this entails in job satisfaction in 395 district pastors of the Brazilian Central Union (UCB), São Paulo, 2017. This research work has a quantitative approach, and uses both a transversal and correlational design. The data collection technique was through the survey, which was directed to the pastors of the UCB.
\end{abstract}

Keywords: Pastoral cycle; burnout syndrome; work satisfaction; pastor

${ }^{\mathrm{a}}$ Correspondencia al autor

E-mail:danielr@upeu.edu.pe 


\section{Introducción}

Uno de los problemas que se observa en las iglesias protestantes desde hace algunas décadas es la alta rotación de pastores. El mismo fenómeno existe también en los distritos misioneros de la IASD en América del Sur. En consecuencia, surgen dificultades que limitan el trabajo del pastor, afectando al crecimiento de la iglesia y causando daños a la familia pastoral. Aunque actualmente el ciclo pastoral a corto plazo (CPCP) sea prevalente en gran parte de iglesias y denominaciones, de acuerdo con Conner (1987) eso "es relativamente un nuevo desarrollo en la historia de la iglesia."

Asimismo, Daniel Rode (1994), es un autor adventista que alerta en cuanto al asunto de la duración del ciclo pastoral (CP) en sus escritos. Él advierte que el promedio de permanencia de los pastores adventistas es muy pequeño, y no llega a alcanzar todas las etapas del CP. Él observa que: "Lamentablemente, el promedio de permanencia de los pastores en sus distritos en toda la América del Sur es sólo de dos años y medio.” De acuerdo con Rode, "las transiciones para nuevos distritos necesitan ser gestionadas teniendo en cuenta el progreso de la iglesia y del pastor como el líder más influente, favoreciendo pastorados largos."

De igual forma, es importante mencionar qué autores adventistas que escribieron sobre el CP destacan la importancia del ciclo pastoral a largo plazo (CPLP). Versteeg (1982) habla de su positiva experiencia de 12 años como pastor de la iglesia de Garden Grove, California. Por su parte, Arnold Kurtz afirma que es necesario que el CP dure más tiempo (1980). R. Edward Turner (1980) también reafirma la necesidad de tener CPLP en las iglesias. Monte Sahlin (1990) destaca la necesidad de la continuidad del liderazgo para lograr mayores resultados. Así también Norman Miles (1990) observa que la tarea del pastor es una obra de larga extensión en el mismo lugar. De acuerdo con Joel Sarli (1981), para que un pastor pueda desarrollar su trabajo apropiadamente no debe ser trasladado de iglesia constantemente. Él afirma que: "La iglesia adventista comprende que el principio de rotación de pastores es beneficioso para la iglesia, pero parece que este principio está siendo usado sin las debidas consideraciones de sus más profundas implicaciones".

Algunas de estas implicaciones tienen que ver con síndrome de burnout y satisfacción laboral. El síndrome de burnout pastoral es real y puede tener relación con la duración del CP. Ludwig (2002) comenta a cerca de un estudio realizado en Indianápolis, 
donde se concluyó que una de las tres principales razones para la salida de pastores del ministerio es el síndrome de burnout.

Por ello, el CPCP puede ser marcado por síndrome de burnout (SB) cuándo hay cambios precoces motivados por falta de apoyo organizacional delante de conflictos con líderes locales en las iglesias. Ante esto, Jetro Ferreira da Silva (2011) comenta que algunos estudios demuestran que hay una correlación entre la falta de apoyo organizacional con el síndrome de burnout. Estos estudios muestran que "cuánto menos apoyo, mayor es la incidencia del síndrome".

Por otro lado, los pastores que desarrollan un CPLP parecen estar más propensos a SB. Conner (1987) enfatiza esta correlación en CPLP al afirmar que: "La constante dádiva de tiempo e energía durante períodos prolongados puede drenar la vitalidad... Se puede producir el agotamiento de un pastor a largo plazo.” Asimismo, Christina Maslach (1977) afirma que uno de los altos riesgos de síndrome de burnout es trabajar estrechamente y intensamente con otras personas durante un período prolongado de tiempo.

En otro orden de cosas, el SB también tiene correlación con la satisfacción laboral (SL). Paul Spector (1997) cita algunos estudios que confirman esta correlación, y afirma que: "el síndrome de burnout se correlaciona con la satisfacción laboral de manera significativa en que los empleados insatisfechos son propensos a reportar altos niveles de sindrome de burnout". Del mismo modo, Cristiane da Rosa y Mary Sandra Cardoso (2005) también destacan las investigaciones que identificaron significativa correlación entre SB y SL.

Sin embargo, las mayores contribuciones sobre el tema del CP fueron escritas por autores inter-denominacionales, que analizaron estos asuntos en un contexto de gobierno eclesiástico congregacional. El sistema congregacionalista "enfatiza el papel del cristiano individual y hace de la congregación el centro de la autoridad, ... El énfasis radica en la autonomía y la independencia de la iglesia local”. Pero, ¿qué significa la autonomía de la iglesia en esta forma de gobierno?

El principio de autonomía significa que cada iglesia es autónoma. Cada congregación llama su propio pastor y determina su presupuesto y sus decisiones no requieren ratificación o aprobación externa. Así, la forma de gobierno eclesiástico congregacional es diferente de la forma de gobierno de la Iglesia Adventista del Séptimo Día (IASD). 
La forma de gobierno de la IASD es representativa. Este modelo reconoce que la autoridad de la iglesia descansa sobre sus miembros y se expresa por medio de representantes debidamente elegidos en cada nivel de la organización, con responsabilidad ejecutiva delegada a entidades y funcionarios para dirigir la iglesia en su nivel.

A pesar de las diferencias de gobierno y remuneración, Zambelli (1997) observa que estas diferencias "no distorsionan la realidad de la tarea pastoral que, en ambos casos, tienen más similitudes que desigualdades." Se cree, por lo tanto, que las conclusiones obtenidas a través de estudios en contexto congregacional pueden ser aplicables al contexto adventista, hasta cierto punto, al llevar en cuenta las similitudes del trabajo pastoral.

George Barna (1997), al comentar sobre la salida de los pastores de las iglesias, explica que algunos de ellos abandonan porque "pueden desanimar a causa de un ambiente hostil’". Y eso lleva a una pregunta crucial: ¿Hay alguna relación entre la duración del CP y la SL de los pastores?

La SL del pastor es un factor importante para tener en cuenta en relación al CP, porque está directamente relacionado con la movilidad. De acuerdo con Evadio Pereira Filho (2014), "La existencia de la relación negativa entre satisfacción laboral y movilidad fue largamente comprobada en estudios empíricos".

Si el pastor no disfruta de esta sensación de equilibrio en un distrito, su voluntad de abordar los problemas a largo plazo se verá amenazada. Eso tiene que ver con expectativas que son nutridas, pero muchas veces no son realizadas. Por eso, el pastor “estará insatisfecho tanto si cree que está en desventaja respecto a sus compañeros, como si considera que el trabajo anterior le ofrecía mejores condiciones".

\section{Método}

La población de esta investigación estuvo conformada por 395 pastores distritales de la Unión Central Brasileira (UCB), São Paulo, 2017. De esta población, se consideró una muestra probabilística de 129 pastores por medio de una fórmula para calcular el tamaño de la muestra. El cálculo fue hecho con un nivel de confianza del 93.35\%, con un error de estimación de 0.0665 . Por ello, el presente trabajo de investigación es de enfoque cuantitativo, y usa un diseño tanto transversal como correlacional. Es cuantitativo dado que los datos son analizados a través de procedimientos estadísticos, y porque se usan teorías y conceptos preconcebidos para determinar los datos que serán recolectados. Es 
transversal porque participan una cantidad significativa de personas en la investigación a la vez, porque se incluye diversas medidas en la misma encuesta y además porque se puede obtener información instantánea sobre las creencias, actitudes y comportamientos actuales de la población en estudio. Además, es de diseño correlacional, por que se recopilan datos para determinar el grado de relación entre dos o más variables. La técnica de recolección de datos fue por medio de la encuesta, la que fue dirigida a pastores de la UCB.

\section{Resultados}

Como se muestra en la tabla 1, de la duración del ciclo pastoral con el síndrome de burnout, se observa que el coeficiente de correlación de Rho Spearman $=-0.090$, y el valor $\mathrm{p}=.351$. A un nivel de significancia de .05 , se cumple que $\mathrm{p}>\alpha$, lo que significa que se acepta la hipótesis nula, que señala que no existe una relación significativa entre la duración del ciclo pastoral y el síndrome de burnout, en los pastores de la UCB, São Paulo, 2017. Además, el resultado del coeficiente de correlación indica que existe una correlación inversa prácticamente nula entre la duración del ciclo pastoral y el síndrome de burnout.

Tabla 1. Correlación de la duración del ciclo pastoral con el síndrome de burnout

\begin{tabular}{llc}
\hline Rho Spearman & & \multicolumn{1}{c}{$\begin{array}{c}\text { Sindrome de } \\
\text { burnout }\end{array}$} \\
\hline \multirow{3}{*}{ Satisfacción laboral } & $\begin{array}{l}\text { Coeficiente de } \\
\text { correlación }\end{array}$ & -.090 \\
\cline { 2 - 3 } & Sig. (bilateral) & .351 \\
\cline { 2 - 3 } & $\mathrm{N}$ & 129 \\
\hline
\end{tabular}

Como se muestra en la tabla 2, de la duración del ciclo pastoral con la satisfacción laboral, se observa que el coeficiente de correlación de Rho Spearman $=-0.220$, y el valor $\mathrm{p}=.023$. A un nivel de significancia de .05 , se cumple que $\mathrm{p}<\alpha$, lo que significa que se rechaza la hipótesis nula y se acepta la hipótesis alternativa, que señala que existe una relación significativa entre la duración del ciclo pastoral y la satisfacción laboral, en los pastores de la UCB, São Paulo, 2017. Además, el resultado del coeficiente de correlación indica que existe una correlación directa baja entre la duración del ciclo pastoral y la satisfacción laboral.

Tabla 2. Correlación de la duración del ciclo pastoral con la satisfacción laboral 


\begin{tabular}{lll}
\hline \multirow{2}{*}{$\begin{array}{l}\text { Duración promedio de ciclo } \\
\text { pastoral }\end{array}$} & $\begin{array}{l}\text { Coeficiente de } \\
\text { correlación }\end{array}$ & .220 \\
\cline { 2 - 3 } & Sig. (bilateral) & .023 \\
\cline { 2 - 3 } & $\mathrm{N}$ & 129 \\
\hline
\end{tabular}

\section{Resultados 1}

En la siguiente tabla 1, se observa que la mayoría de pastores distritales que participan en la investigación pertenecen a la Asociación Paulista do Vale (20.1\%); con respecto al estado civil, se puede apreciar que el $99.2 \%$ de pastores distritales son casados, existiendo solo un pastor divorciado que representa el $0.8 \%$; se observa también que el 92.2\% tienen algún hijo; en relación al rango de edad se aprecia que la tercera parte de pastores distritales tienen entre 31 y 40 años de edad (33.3); el 62\% de pastores cuenta con estudios de posgrado; y el 90.7\% ya son pastores ordenados. Por otro lado, el $55.1 \%$ de pastores tienen entre 11 y 30 años como distrital; con respecto al tiempo que permanecieron en su último distrito, más de la mitad (54.3\%) respondieron que estuvieron entre 3 y 4 años; el $34.9 \%$ de pastores ya tuvieron entre 3 y 5 cambios de distrito; el $60.5 \%$ no fueron cambiados en el último año; y el 51.2\% no llevan más de 10 años en la UCB. Un punto importante es que el $71.3 \%$ de pastores distritales afirmaron que el tiempo promedio que permanecen en un distrito (ciclo pastoral) es entre 3 y 4 años.

Tabla 3. Análisis de frecuencia de las características de los pastores de la UCB

\begin{tabular}{ccc}
\hline & $\boldsymbol{N}$ & $\boldsymbol{\%}$ \\
\hline Asociación & & \\
\hline Asociación Paulista do Vale & 26 & 20.2 \\
Asociación Paulista Leste & 19 & 14.7 \\
Asociación Paulistana & 11 & 8.5 \\
Asociación Paulista Sudeste & 11 & 8.5 \\
Asociación Paulista Sudoeste & 14 & 10.9 \\
Asociación Paulista Central & 12 & 9.3 \\
Asociación Paulista Oeste & 16 & 12.4 \\
Asociación Paulista Sul & 20 & 15.5 \\
\hline Estado civil & & \\
\hline Casado & 128 & 99.2 \\
Divorciado & 1 & 0.8 \\
\hline Tiene hijos Si & 119 & \\
\hline$\quad$ No & 10 & 7.8 \\
\hline Edad 25-30 años & 6 & 4.7
\end{tabular}




\begin{tabular}{|c|c|c|}
\hline 31-40 años & 43 & 33.3 \\
\hline $41-50$ años & 40 & 31.0 \\
\hline 51-60 años & 36 & 27.9 \\
\hline 61-65 años & 4 & 3.1 \\
\hline \multicolumn{3}{|l|}{ Educación } \\
\hline Superior completo & 49 & 38 \\
\hline Posgrado & 80 & 62 \\
\hline \multicolumn{3}{|c|}{ Ordenado al ministerio pastoral } \\
\hline $\mathrm{Si}$ & 117 & 90.7 \\
\hline No & 12 & 9.3 \\
\hline \multicolumn{3}{|c|}{ Tiempo como pastor distrital } \\
\hline 0-4 años & 21 & 16.3 \\
\hline 5-10 años & 28 & 21.7 \\
\hline $11-20$ años & 37 & 28.7 \\
\hline 21-30 años & 34 & 26.4 \\
\hline 31-35 años & 9 & 7.0 \\
\hline \multicolumn{3}{|l|}{ Tiempo en último distrito } \\
\hline $1-2$ años & 31 & 24 \\
\hline 3-4 años & 70 & 54.3 \\
\hline 5-6 años & 23 & 17.8 \\
\hline 7-8 años & 5 & 3.9 \\
\hline \multicolumn{3}{|c|}{ Número de cambios distritales } \\
\hline $1-2$ cambios & 37 & 28.7 \\
\hline 3-5 cambios & 45 & 34.9 \\
\hline 6-8 cambios & 33 & 25.6 \\
\hline 9-11 cambios & 11 & 8.5 \\
\hline 12-14 cambios & 3 & 2.3 \\
\hline \multicolumn{3}{|l|}{$\begin{array}{l}\text { Duración promedio de ciclo } \\
\text { pastoral }\end{array}$} \\
\hline 1 año & 10 & 7.8 \\
\hline 2 años & 17 & 13.2 \\
\hline 3 años & 53 & 41.1 \\
\hline 4 años & 39 & 30.2 \\
\hline 5 años & 8 & 6.2 \\
\hline 6 años & 2 & 1.6 \\
\hline \multicolumn{3}{|c|}{ Cambiado en el último año } \\
\hline $\mathrm{Si}$ & 51 & 39.5 \\
\hline No & 78 & 60.5 \\
\hline \multicolumn{3}{|c|}{ Tiempo de distrital en UCB } \\
\hline $0-4$ años & 30 & 23.3 \\
\hline 5-10 años & 36 & 27.9 \\
\hline 11-14 años & 14 & 10.9 \\
\hline 15-19 años & 20 & 15.5 \\
\hline 20-24 años & 12 & 9.3 \\
\hline 25-29 años & 11 & 8.5 \\
\hline Mas de 30 & 6 & 4.7 \\
\hline
\end{tabular}




\section{Resultados 2}

Como se muestra en la tabla 4, a pesar que la relación chi-cuadrado/grados de libertad tiene un valor de 2.105, que al estar por debajo de 3 puede considerarse aceptable, los índices de ajuste obtenidos RMSEA=0.093, TLI=0.703, CFI=0.717 y PNFI=0.548. Además, se cumple que el RMSEA $>\alpha$, lo que significa que se acepta la hipótesis nula; por lo tanto, no existe una relación significativa entre la duración del ciclo pastoral, síndrome de burnout y satisfacción laboral, en los pastores de la UCB, São Paulo, 2017.

Tabla 4. Correlación de la duración del ciclo pastoral, sindrome de burnout y satisfacción laboral. Resultados en el software AMOS

\begin{tabular}{|c|c|c|c|c|c|}
\hline \multicolumn{6}{|c|}{ CMIN } \\
\hline Model & NPAR & CMIN & $\mathrm{DF}$ & $\mathrm{P}$ & CMIN/DF \\
\hline Default model & 92 & 1984.804 & 943 & .000 & 2.105 \\
\hline \multicolumn{6}{|c|}{ Baseline Comparisons } \\
\hline \multirow{2}{*}{ Model } & NFI & RFI & IFI & TLI & \multirow{2}{*}{ CFI } \\
\hline & Delta1 & rho1 & Delta2 & rho2 & \\
\hline Default model & 0.575 & 0.554 & 0.720 & 0.703 & 0.717 \\
\hline \multicolumn{6}{|c|}{ Parsimony-Adjusted Measures } \\
\hline Model & PRATIO & & PNFI & & PCFI \\
\hline Default model & 0.953 & & 0.548 & & 0.683 \\
\hline \multicolumn{6}{|c|}{ RMSEA } \\
\hline Model & RMSEA & LO 90 & HI 90 & & PCLOSE \\
\hline Default model & 0.093 & 0.087 & 0.099 & & 0.000 \\
\hline
\end{tabular}

\section{Resultados 3}

Como se muestra en la tabla 5, de la duración del ciclo pastoral con la satisfacción laboral, se observa que el coeficiente de correlación de Rho Spearman $=-0.220$, y el valor $\mathrm{p}=.023$. A un nivel de significancia de .05 , se cumple que $\mathrm{p}<\alpha$, lo que significa que se rechaza la hipótesis nula y se acepta la hipótesis alternativa, que señala que existe una relación significativa entre la duración del ciclo pastoral y la satisfacción laboral, en los pastores de la UCB, São Paulo, 2017. Además, el resultado del coeficiente de correlación indica que existe una correlación directa baja entre la duración del ciclo pastoral y la satisfacción laboral.

Tabla 5. Correlación de la duración del ciclo pastoral con la satisfacción laboral 


\begin{tabular}{lll}
\hline \multirow{2}{*}{$\begin{array}{l}\text { Duración promedio de ciclo } \\
\text { pastoral }\end{array}$} & $\begin{array}{l}\text { Coeficiente de } \\
\text { correlación }\end{array}$ & .220 \\
\cline { 2 - 3 } & Sig. (bilateral) & .023 \\
\cline { 2 - 3 } & $\mathrm{N}$ & 129 \\
\hline
\end{tabular}

\section{Resultados 4}

Como se muestra en la tabla 6 , del síndrome de burnout con la satisfacción laboral, se observa que el coeficiente de correlación de Rho Spearman $=-0.480$, y el valor $\mathrm{p}=$ .000 . A un nivel de significancia de .05 , se cumple que $p<\alpha$, lo que significa que se rechaza la hipótesis nula y se acepta la hipótesis alternativa, que señala que existe una relación significativa entre el síndrome de burnout y la satisfacción laboral, en los pastores de la UCB, São Paulo, 2017. Además, el resultado del coeficiente de correlación indica que existe una correlación inversa moderada entre el síndrome de burnout y la satisfacción laboral.

Tabla 18. Correlación del síndrome de burnout con la satisfacción laboral

\begin{tabular}{llc}
\hline Rho Spearman & & Sindrome de burnout \\
\hline \multirow{3}{*}{ Satisfacción laboral } & $\begin{array}{l}\text { Coeficiente de } \\
\text { correlación }\end{array}$ & -.480 \\
\cline { 2 - 3 } & Sig. (bilateral) & .000 \\
\cline { 2 - 3 } & $\mathrm{N}$ & 129 \\
\hline
\end{tabular}

\section{Conclusión}

De acuerdo a los resultados presentados en esta investigación, se concluye:

1. No existe una relación significativa entre la duración del ciclo pastoral, sindrome de burnout y satisfacción laboral, en los pastores de la Unión Central Brasileira, São Paulo, dado que el RMSEA $=.093$, y sus demás índices de ajuste son TLI $=.703$, $\mathrm{CFI}=.717$ y $\mathrm{PNFI}=.548$.

2. No existe una relación significativa entre la duración del ciclo pastoral y el sindrome de burnout, en los pastores de la Unión Central Brasileira, São Paulo, dado que el $\mathrm{p}=.351$, y su coeficiente de correlación es -.090.

3. Existe una relación significativa entre la duración del ciclo pastoral y la satisfacción laboral, en los pastores de la Unión Central Brasileira, São Paulo, dado que el $p=.023, y$ su coeficiente de correlación es .220. 
4. Existe una relación significativa entre el síndrome de burnout y la satisfacción laboral, en los pastores de la Unión Central Brasileira, São Paulo, dado que el $p=.000 \mathrm{y}$ su coeficiente de correlación es -.480.

\section{Referencias}

Abdala, Emílio. "Reacendendo uma paixão antiga". http://aletheiaasd.blogspot. com.br/2011/02/plantio-de-igrejas-iii.html (consultado: 13 de septiembre, 2016).

Acosta, J. "Dirigir: delegar, motivar, comunicar, delegar, dirigir reuniones". Madrid: ESIC Editorial, 2013. Citado en: Esteban Silva, Felipe Reynaldo. "Cultura organizacional, compromiso cristiano y la satisfacción laboral en los docentes de la Universidad Peruana Unión de la ciudad de Lima, Peru, 2013”. Tesis doctoral, Universidad Peruana Unión, 2013.

Adams, Candace Sue. "The Significance of Sustaining Clergy Spiritual Wellness During Pastoral Transitions". Tesis doctoral, University of Dubuque Theological Seminary, 2007.

Anderson, Lynn. "Why I've Stayed". Leadership Journal 7. no. 3. Summer 1986: 76-82. http://www.christianitytoday.com/pastors/1986/summer/8613076.html (consultado: 1 de diciembre, 2016).

Anderson, Cara J. "Pastoral Sindrome de burnout and Marital Satisfaction". Tesis doctoral, Fuller Theological Seminary, 2010.

Anderson, Carole Brousson. "The Experience of Growing Up in a Minister's Home and the Religious Commitment of the Adult Child of a Minister". Tesis doctoral, Andrews University, 1995.

Arn, Charles. "Pastoral Longevity and Church Growth". Enrichment Journal (spring, 2014).

http://enrichmentjournal.ag.org/201402/201402_001_016_Pastoral_Longevity.cf m / (consultado: 16 de enero de 2017).

Arrais, Jonas. Procura-se um bom pastor. Tatuí, SP: Casa Publicadora Brasileira, 2011.

Baker, David Weston, Alexander, Thomas Desmond, y Sturz, Richard J. Obadias, Jonas, Miquéias, Naum, Habacuque e Sofonias: introdução e comentário. Vol. 23. Cultura Bíblica. São Paulo, SP: Vida Nova, 2001.

Barna, George. Leaders on Leadership: Wisdom, Advice and Encouragement on the Art of Leading God's People. Ventura, CA: Regal Books, 1997. 
.The habits of Highly Effective Churches: Being Strategic in your God-Given Ministry. Grand Rapids, MI: Baker Books, 2014. Kindle edition. . The Second Comming of the Church. Nashville, TN: Word Publishing, 1998. . Today's Pastors: A Revelating Look at What Pastors Are Saying About Themselves, They Peers and the Pressures They Face. Oxnard, CA: Regal Books, 1993.

Barro, Jorge Henrique. "Moisés, líder e libertador: um estudo de caso da liderança pastoral de Moisés - seu chamado, suas crises e sua humanidade”. Revista Batista Pioneira 4, no. 1 (junio, 2015): 137-159. http://revista.batistapioneira.edu.br/index.php/rbp (consultado: 16 de junio de 2017).

Bauman, Zygmunt. Vida para consumo: a transformação das pessoas em mercadorias. Rio de Janeiro, RJ: Jorge Zahar Editor, 2008.

Benedito, Marian, Bonavia, Tomás y Llinares, Lucía. "Relación entre las prioridades de valor y satisfacción laboral". Revista colombiana de psicología, no. 17 (2008): $59-74$.

Bevilacqua, Lidiane Amanda, da Silva, Marcelo Cozzensa, Reichert, Felipe Fossati, y Rombaldi, Airton José. “Qualidade de vida e satisfação com o trabalho de profissionais das academias de ginástica da cidade de Pelotas/RS”. Revista brasileira de atividade física e saúde 19, no 3, (2014): 314-324, DOI: http://dx.doi.org/10.12820/rbafs.v.19n3p314, (consultado: 6 de septiembre, 2016).

Bluedorn, Allen C. "A Unified Model of Turnover from Organizations". Human Relations 35, (February 1982): 135-153.

Bonthrone, P. J. "Job Stress Blamed for Clergy "Burn-out"”. The Telegraph (25 de setiembre, 2000). http://www.telegraph.co.uk/news/uknews/1356674/Job-stressblamed-for-clergy-burn-out.html (consultado: 24 de abril de 2017).

Branson, Roy. Church Split. Bristol, TN: Landmark Publications, 1990. citado en: Brown, Richard W. Restoring the Vow of Stability: the Keys to Pastoral Longevity. Camp Hill, PA: Christian Publications, 1993

Bromley, Jeffrey. ed. "The International Standard Bible Encyclopedia”. 4 Vols. Grand Rapids, MI: Eardmans, 1982. Citado en: Loomis, Gregory Mark "Key Factors of Pastoral Longevity in the Evangelical Free Church of America". Tesis doctoral, Trinity Evangelical Divinity School, 2006. En Tren Dissertations. Paper 3256. 
http://place.asburyseminary.edu/ trendissertations/3256. (consultado: 15 de junio, 2017).

Brown, Richard W. Restoring the Vow of Stability: the Keys to Pastoral Longevity. Camp Hill, PA: Christian Publications, 1993.

Brubaker, David R. "Change and Conflict in Congregations: Examining the Effects of Change on Conflict in Religious Congregations". Tesis doctoral, University of Arizona, 2007.

Brunner, Emil. The Word and the World. Lexington, KY: American Theological Lybrary Association, 1965.

Burrill, Russell. Revolução na Igreja: com o incrivel poder do ministério leigo. Almargem do Bispo, Portugal: Publicadora Atlântico, S.A., 1999.

Byrd, T., Cochran, J., Silverman, I., \& Blount, W. "Behind Bars: an Assessment of the Effects of Job Satisfaction, Job-Related Stress, and Anxiety of Jail Employees' Inclinations to Quit”. Journal of Crime and Criminal Justice 23, (2000): 69-89.

Chiang, M., Martin, J., y Núñes, A.“Relaciones entre el clima organizacional y la satisfacción laboral". Madrid: Pontificia Universidad Comillas, 2010. Citado en Silva, Felipe Reynaldo Esteban. "Cultura organizacional, compromiso cristiano y la satisfacción laboral en los docentes de la Universidad Peruana Unión de la ciudad de Lima, Peru, 2013”. Tesis doctoral, Universidad Peruana Unión, 2013.

Chng, Alex Peng Lian. "The Impact of Sindrome de burnout and Spiritual Well-Being on the Job Satisfaction of Pastors within the Methodist Church in Singapore". Tesis doctoral, Asbury Theological Seminary, 2011.

Cionca, John R. Red Light, Green Light: Discerning the Time for a Change in Ministry. Grand Rapids, MI: Baker Books, 1994.

Cloud, Henry. Necessary Endings: The Employees, Businesses, and Relationships that All of Us Have to Give Up in Order to Move Forward. New York, NY: Harper Collins Publishers, 2010.

Colcord, W. A. New York Independent. October 1891. Citado en: Hudson, Stan. Pastoral Roles in Adventism's First Century. Ministry Magazine, junio de 1997. https://www.ministrymagazine.org/archive/1997/06/pastoral-roles-inadventisms-first-century. (consultado: 13 de septiembre, 2016).

Conner, R. Dwaine. Called to Saty: Keys to a Longer Pastorate in a Southern Baptist Church. Nashville, TN: Convention Press, 1987. 
Daniells, Arthur G. The Church and Ministry. Riverside, Jamaica: Watchman Press, 1912. Citado en: Fowler, John. Adventist Pastoral Ministry. Boise, Idaho:Pacific Press Pub. Assn., 1990.

Danielson, Richard Allen, "Beating the Odds: Succesfully Following a Long-Term Pastor". Tesis doctoral, Asbury Theological Seminary, 2001.

Dederen, Raul. Tratado de teologia: adventista do sétimo dia. Tatuí, SP: Casa Publicadora Brasileira, 2011.

Din, Young Sun. "A Study of Pastoral Sindrome de burnout Among Korean-American Pastors". Tesis doctoral, Liberty Theological Seminary, 2009.

Dinis, Liliana Isabel Correia, y Fronteira, Inês, “A influência da rotação no trabalho na satisfação profissional dos enfermeiros num serviço de cirurgia”, Revista de Enfermagem Referência IV, no. 5, (2015): 19. http://dx.doi.org/10.12707/RIV14008. (consultado: 30 de agosto, 2016).

Dudley, Roger L. y Kilsher, Carole Lucke. "A New View of the Pastor's Wife”. Ministry Magazine (june, 1981): 28.

https://www.ministrymagazine.org/archive/1981/06/a-new-view-of-the-pastorswife (consultado: 20 de septiembre, 2016). , Roger, y Swanson, H. Peter. "What Makes a Pastor Effective?". Ministry (diciembre, 2000): 26-29. https://www.ministrymagazine.org/archive/2000/12/what-makes-a-pastoreffective (consultado: 15 de mayo de 2017).

Dusilek, Nancy Gonçalves. Mulher sem nome: dilemas e alternativas da esposa do pastor. São Paulo: Edtora Vida, 1996.

Erickson, Millard J. Teologia sistemática. São Paulo: Vida Nova, 2015.

Esteban Silva, Felipe Reynaldo. "Cultura organizacional, compromisso cristiano y la satisfacción laboral en los docentes de la Universidad Peruana Unión de la ciudad de Lima, Peru, 2013”. Tesis doctoral, Universidad Peruana Unión, 2013.

Exantus, Ruben. Pastoral Sindrome de burnout and Leadership Styles. Bloomington, IN: Author House, 2012.

Exley, Richard. Perigos que rondam o ministério: tragédias que podem ser evitadas. Artur Nogueira, SP: Centro Adventista de Artes Gráficas - UCB, 2003.

Farris, Lawrence W. Ten Commandments for Pastors New to a Congregation. Grand Rapids, MI: William B. Eerdmans Publishing Company, 2003. 
Faulkner, Brooks R. Sindrome de burnout in Ministry. Nashville, Broakman Press, 1981. citado en: Williams, Ivan L. "Pastoral Sindrome de burnout and Renewal in Seventh-Day Adventist Ministry". Profesional Project, Claremont School of Theology, 2002.

Ferreira Silva, Jetro. "O sindrome de burnout pastoral na perspectiva da teologia prática: definições, causas e prevenção". Tesis doctoral, Pontífica Faculdade de Teologia Nossa Senhora da Assunção, São Paulo, 2006.

Fickel, Julie. "Sindrome de burnout in Pastors and Church Leadership". M1 Psychology. http://mlpsychology.com/sindrome de burnout-in-pastors-andchurch-leadership/ (consultado: 29 de marzo de 2017).

Filho, E. P., Florencio, C. C. A., y Leone, N. M. C. P. G.. "Satisfação profissional e cultura organizacional: um estudo de caso em uma instituição federal de ensino" Holos 4, (agosto 2014): 344-360.

Friedman, Edwin. Generation to Generation: Family Process in Church and Synagogue. New York, London: The Guilford Press, 2011. Kindle Edition.

Finzel, Hans. The Top Ten Mistakes Leaders Make. Wheaton, IL: Victor Books, 1994. Fowler, J. W. Adventist Pastoral Ministry, 1ra. ed. Boise: Pacific Press Publishing Association, 1990.

Gall, Meredith D., Joyce P. Gall y Walter R. Borg. Educational Research: An introduction. 7ma ed. New York: Pearson Education, 2003.

Gay, L. R., Geoffrey E. Mills y Peter W. Airasian. Educational Research: Competencies for analysis and applications. 10ma ed. New York: Pearson, 2012.

George, Denise. What Pastors Wish Church Members Knew: Helping People Understanding the Needs, Fears, and Challenges of Church Leaders Today. Grand Rapids, MI: Zondervan, 2009. Kindle Editon.

Gil-Monte, Pedro R., y Peiró, José M. “A Study on Significant Sources of the "Sindrome de burnout Syndrome" in Workers at Occupational Centers for the Mentally Disabled" Psychology in Spain 1, no. 1 (1997): 55-62. Citado en: Ferreira da Silva, Jetro. "O sindrome de burnout pastoral na perspectiva da teologia prática: definições, causas e prevenção". Tesis doctoral, Pontífica Faculdade de Teologia Nossa Senhora da Assunção, São Paulo, 2006.

Gilmore, Thomas North. Making a Leadership Change: How Organizations Can Handle Leadership Transitions Successfully. Lincoln, NE: iUniverse, 2003. 
Giorgiov, Adrian Dorin. "The Effect Accoutability on Pastoral Stress and Sindrome de burnout Among Select Hungarian Baptist Pastors”. Tesis doctoral, Southwestern Baptist Theological Seminary, 2002.

Gomes de Deus, Persio Ribeiro. “As influências do sentimento religioso sobre o cristão portador de depressão”. Tesis de maestria, Universidade Presbiteriana Mackenzie, 2008.

http://www.dominiopublico.gov.br/download/texto/cp061758.pdf (consultado: 03 de maio de 2017).

Gonçalves, Kléber de Oliveira. "A Critique of the Urban Mission of the Church in the Light of Emerging Postmodern”. Tesis Doctoral, Andrews University, 2005.

Gonçalves, Tatiana "Satisfação profisssional e percepção do apoio organizacional: estudo exploratório como contributo para um sistema de compensações e benefícios no setor farmacêutico". Tesis de maestría, Universidad de Lisboa, 2011.

Gotuzzo, Raúl Pino. Metodologia de la investigación. Lima: Editorial San Marcos, 2006.

Green, Russell Brian. "Stay the Course: The Necessity of a Long Pastoral Tenure for Maximum Church Effectiveness". Tesis doctoral, Biola University, 2002.

Grubbs, Bruce. The First Two Years: a Pastor's Guide to Getting Started in a Church. Cleveland: Pathway Press, 1979.

Guerrero, Abraham. Escape de la mecedora: rompiendo el ciclo de oscilación entre el éxito y el fracaso en tu vida personal y en la iglesia. Kissimee, FL: Triunfo Publicaciones, 2014.

Guerrero, Saul Barcelo. "Un programa de renovación vocacional para los pastores de la Asociación de Baja California, México”. Tesis doctoral, Andrews University, 2011. En Digital Commons @Andrews University, http://digitalcommons.andrews.edu/cgi/viewcontent.cgi?article $=1011 \&$ context $=$ dmin (consultado: 24 de abril de 2017).

Hadaway, C. kirk. Church Growth Principles: Separating Fact from Fiction. Nashville, TN: Broadman Press, 1991.

Hamilton, Ronald Ernest. "The Development of an Effective Long-Term Pastorate". Tesis doctoral, Fuller Theological Seminary, 1999. 
Harison, Paul V. "Pastoral Turnover and the Call to Preach" JETTS 44:1 March 2001: 87-105. http://www.mattblackmon.com/pyne/turnover.pdf. (consultado: 5 de setiembre, 2016).

Hauerwas, Stanley, y Willimon, William H. "Ministry is More Than a Helping Profession". The Christian Century. (15 de marzo, 1989): 282-284. http://eds.a.ebscohost.com/eds/pdfviewer/pdfviewer?sid=46dcf9f8-c099-48848c0d-6a29b9c28ca3\%40sessionmgr4007\&vid=1\&hid=4202 (consultado: 26 de abril de 2017).

Hernández, Claudia Alejandra "La motivación y satisfacción laboral de los docentes en cuatro escuelas de enseñanza media en México". Tesis doctoral, Universidad Autónoma Metropolitana Unidad Xochimilco, 2010.

Herzberg, F., Mausner, B., \& Snyderman, B. the Motivation to Work. New York: 1959. Citado en: Gonçalves, Tatiana "Satisfação profissional e percepção do apoio organizacional: estudo exploratório como contributo para um sistema de compensações e benefícios no setor farmacêutico". Tesis de maestria, Universidade de Lisboa, 2011.

Hichs, Donald. "A Study of the Conflicts within Churches that Lead to the Termination of Pastors within the Southern Baptist Convention, Accompanied by a Proposal of Preventive and Interventional Solutions". Tesis Doctoral, Liberty Baptist Theological Seminary, 2010.

http://digitalcommons.liberty.edu/cgi/viewcontent.cgi?article $=1428 \& \operatorname{context}=\mathrm{do}$ ctoral (consultado: 20 de septiembre, 2016).

Ho, W. H., Chang, C. S., Shih, Y. L., \& Liang, R. D. Effects of Job Rotation and Role Stress Among Nurses on Job Satisfaction and Organizational Commitment, 2009. Recuperado de http://www.biomedcentral.com/1472-6963/9/8 doi:10.1186/1472-6963-9-8. Citado en Dinis, Liliana Isabel Correa, y Fronteira, Inês "A influência da rotação no trabalho na satisfação profissional dos enfermeiros num serviço de cirurgia" Revista de enfermagem referência IV, no. 5, (2015): 18. http://dx.doi.org/10.12707/RIV14008. (consultado: 31 de agosto, 2016).

Hobgood, William. "The Long-Tenured Pastorate: A Study of Ways to Build Trust". Tesis doctoral, Lancaster (PA) Seminary, 1982. Citado en: Brown, 68. Hoppock, R. Job Satisfaction. Harperand Brothers: New York, 1935. 
Horne, Chevis F. "Forty Years in The Same Pulpit: What Makes for Long Pastorates". Macon, GA: Smyth \& Helwys, Publishing, 1995.

Hoskins, John H. "Obeying God's Call for the Long Haul: a Study of Pastoral

Longevity and Tenure Among Independent Baptist and Bible Pastors in West Virginia". Tesis doctoral, Temple Baptist Seminary, 2000.

Hovater, Steven. Long-Term Sermon Planning: The Craft of Ministry Project Book 1. Kindle edition.

Hudson, Stanley A. "Pastoral Roles in Adventism's First Century". Ministry Magazine. (junio de 1997): 10-11.

https://www.andrews.edu/library/car/cardigital/Periodicals/Ministry_Magazine/1 997/1997_06.pdf(consultado: 1 de diciembre, 2016).

Hughes, Kent y Barbara. Liberating Ministry from the Success Syndrome. Wheaton, IL: Tyndale House Publishers, 1987.

Hughes, Wiley E. "Assessing the Causes of Short-Term Pastorates in the North Carolina Conference of the Internacional Pentecostal Holiness Church". Tesis doctoral, Oral Roberts University, 2013.

Hugo, Jorge Allan Iuorno. "La satisfacción laboral y el desgaste emocional de los pastores distritales de la Unión Austral". Tesis doctoral, Universidad Peruana Unión, 2007.

Hull, Bill. The Disciple-Making Pastor: Leading Others in the Journey of Faith. Revised and Expanded Edition. Grand Rapids, MI: Baker Books, 2007. Jaturanonda, C., Nanthavanij, S., \& Chongphaisal, P. "A Survey Study on Weights of Decision Criteria for Job Rotation in Thailand: Comparison Between Public and Private Sectors" International Journal of Human Resource Management 17, no. 10, 2006: 1834-1851. Citado en Dinis, Liliana Isabel Correa, y Fronteira, Inês "A influência da rotação no trabalho na satisfação profissional dos enfermeiros num serviço de cirurgia" Revista de enfermagem referência IV, no. 5, (2015): 18. http://dx.doi.org/10.12707/RIV14008. (consultado: 31 de agosto, 2016). Johnson, John E. "The Old Testament Offices as Paradigm for Pastoral Identity". Biblioteca Sacra 152 (april-june, 1995): 182-200. http://www.galaxie.com/article/bsac152-606-04 (consultado: 14 de junio de 2017).

Júnior, H. B. London, y Wiseman, Neil B. Seu pastor é uma espécie em extinção. São Paulo: Eclesia, 1998. 
, H. B. y Wiseman, Neil B. The Heart of a Great Pastor. Grand Rapids, MI:

Baker Books, 2014. Kindle edition.

Júnior, José Cerqueira Barros. “Avaliação de satisfação no trabalho em mineradora brasileira". Tesis de maestria, Universidade de Franca, 2009.

, Cesário, Manuel, Morraye, Mônica de Andrade, y Júnior, José Dionísio de Paula

"Avaliação de satisfação no trabalho em uma mineradora brasileira",

Investigação 11, (2011): 29-33.

http://publicacoes.unifran.br/index.php/investigacao/ article/view/501/404, (consultado: 6 de septiembre, 2016).

Kemp, Jaime. Pastores em Perigo: ajuda para o pastor, esperança para a igreja. São Paulo: Editora Sepal, 1996.

Kisslinger, Stephen A. "Sindrome de burnout in Presbyterian Clergy of Southwestern Pensylvania”. Tesis doctoral, Indiana University of Pennsylvania, 2007.

Ko, Min Jae. "Development of a Ministry of Caring and Healing to Avoid Pastoral Sindrome de burnout”. Tesis doctoral, Regent University, 2003.

Kurtz, Arnold. "Short-Term Pastorates". Ministry (january 1980): 14-15. https://gcminrnr.s3.amazonaws.com/cdn/ministrymagazine.org/issues/1980/issues/MIN198001.pdf (consultado: 01 de diciembre, 2016).

LaRue, John. “Pastoral Tenure: Are You Church-Hopping?”, CT\&T 11 agosto 1995.

Citado en Zambelli, Ricardo N. "Implicaciones de la duración de los pastorados en la Argentina". Tesis doctoral, Universidad Adventista del Plata, 1997.

Leliévre, Mateo. John Wesley: sua vida e obra. São Paulo: Editora Vida, 1997.

Lipie, Leslie A., y Stinchcomb, Jeanne B. "Should I Stay? Or Should I Go? Job

Satisfaction and Turnover Intent of Jail Staff Throughtout the United States"

Criminal Justice Review 38, no. 2, 229.

Little, Todd D. ed. The Oxford Handbook of Quantitative Methods: Foundations. Vol.

1. New York: Oxford University Press, 2013.

Lohnes, Peter George, "Leading While Leaving: The Role of a Departing Pastor in

Preparing a Church for a Healthy Pastoral Transition”. Tesis doctoral, Acadia Divinity College, 2008.

Locke, E. A. The Nature and Causes of Job Satisfaction, En M. D. Dunnette (Org.). Handbook of Industrial and Organizational Psychology. Chicago: Rand McNally College Publishing Company, 1976, 1297-135. Citado en Gonçalves, Tatiana. "Satisfação profissional e percepção do apoio organizacional: estudo 
exploratório como contributo para um sistema de compensações e benefícios no setor farmacêutico". Tesis de maestria, Universidade de Lisboa, 2011.

Loomis, Gregory Mark "Key Factors of Pastoral Longevity in the Evangelical Free Church of America". Tesis doctoral, Trinity Evangelical Divinity School, 2006. En Tren Dissertations. Paper 3256. http://place.asburyseminary.edu/ trendissertations/3256. (consultado: 9 de Agosto, 2016).

Lopez, M. P. S., y Esteves, M. A. Q. "Relaciones entre satisfacción familiar y laboral: variables moduladoras". Anales de psicología (1995): 63-75.

Ludwig, Glenn E. In it for the Long Haul: Building Effective Long-Term Pastorates. Winsconsin: The Alban Institute, 2002.

Lutzer, Erwin. De pastor para pastor: respostas concretas para os problemas e desafios do ministério. São Paulo: Editora Vida, 2000.

Macedo, Alicia. Estamos de mudança: um guia para famílias em mudança de casa, de cidade, de país. Viçosa, MG: Ultimato, 2011.

Marqueze, Elaine Cristina, y Moreno, Claudia Roberta de Castro, "Satisfação no trabalho e capacidade para o trabalho entre docentes universitários". Psicologia em estudo 14, no 1, (2009): 75-82. http://www.scielo.br/pdf/pe/v14n1/a10v14n1. (consultado: 06 de septiembre, 2016).

Martinez, Maria Carmen “As relações entre a satisfação com aspectos psicossociais no trabalho e a saúde do trabalhador". Tesis de maestria, Universidade de São Paulo, 2002.

Maslach, Christina, y Pines, Ayala. "The Bur-Out Syndrome in the Day Care Setting". Child Care Quarterly 6 (Summer, 1977): 100-113. file://C:/Users/Gerson/Downloads/The_burnout_syndrome_in_the_day_care_se.pdf. (consultado: 04 de enero, 2017). , Christina, Jackson, S. E. “The Measurement of Experienced Sindrome de burnout", Journal of Ocuppational Behavior (1981): 99-113. Citado en: Lima, Carla Fernanda, Oliveira, José Arimatés de, Silva, Élido Santiago da, y Emérito, Antonio de Pádua. "Avaliação psicométrica do Maslach Sindrome de burnout Inventory em profissionais de enfermagem". $2^{\circ}$ Encontro de Gestão de Pessoas e Relações de Trabalho (diciembre, 2009): 1-11.

McIntosh, Gary L. There's Hope for Your Church: Firsts Steps to Restoring Health and Growth. Grand Rapids: Baker Books, 2012. 
, Gary. "Long Pastoral Tenure Can Hurt Your Church". A Blog by the Faculty of Talbot School of Theology. (14 de junio, 2013),

http://www.thegoodbookblog.com/2013/jun/14/long-pastoral-tenure-can-hurtyour-church/ (consultado: 04 de octubre, 2016).

Mead, Loren B. Critical Moment of Ministry: A Change of Pastors. Bethesda, MD: The Alban Institute, 1986

Miles, Norman K.. "The Minister as a Pastor". Ministry Magazine, july 1990: 14. https://www.ministrymagazine.org/archive/1990/07/the-minister-as-a-pastor. (consultado: 21 de enero, 2016).

Mobley, W. H., Griffeth, R. W., Hand, H. H., \& Meglino, B. M. "Review and Conceptual Analysis of the Employee Turnover Process". Psychological Bulletin 86, 1979: 493-522.

Morris, Michael Lane, y Blanton, Priscilla White. "The influence of Work-Related Stressors on Clergy Husbands and Their Wives”. Family Relations 43, no. 2 (Abril, 1994): 189-195. https://www.researchgate.net/publication/271697221, (consultado: 08 de mayo de 2017).

Muck, Terry, ed.. “Shepards Who Have Stayed”. Leadership 4, no. 4 (Fall 1983): 131144. http://www.christianitytoday.com/pastors/1983/fall/8314131.html (consultado: 6 de mayo de 2017).

Nichol, Francis D., ed. Comentário bíblico adventista. 8 Vols. Traducido por Rosangela Lira et. al. Tatuí, SP: Casa Publicadora Brasileira, 2011-2016.

Oswald, Roy M., Hinand, Gail D., Hobgood, William C., y Lloyd, Barton M., New Visions for the Long Pastorate. Bethesda, MD: Alban Institute, 1990.

Oswald, Roy M., The Pastor as Newcomer. Washington, DC: The Alban Institute, 1990.

Pagani, César Luís, trad. Guia para ministros adventistas do sétimo dia. Tatuí, SP: Casa Publicadora Brasileira, 2010.

Pearson, Stewart, "Keys to a Long Pastorate”. Leadership Journal 5, no. 2, (spring 1984): 135-136.

http://www.christianitytoday.com/pastors/1984/spring/8412135.html (consultado: 1 de diciembre, 2016).

Pereyra, Mario. Psicologia da reconciliação: técnicas e estratégias: como reparar os vínculos perdidos. Artur Nogueira, SP: Editora CEDISAU, 1997. 
Perkins-Reed, M. A. Guia para a satisfação no trabalho: um manual da nova era para o sucesso pessoal e profissional. São Paulo: Editora Best Seller, 1990.

Rainer, Thom S. Autopsy of a Deacesed Church: 12 Ways to Keep Yours Alive. Nashville, TN: B\&H Publishing, 2014. , Thom S. "Seven Reasons Pastors Burn Out”. 28 de septiembre, 2013. bajo Thom S. Rainer Growing Healthy Churches. Together. http://thomrainer.com/2013/09/seven-reasons-pastors-burn-out/ (consultado: 27 de março de 2017). . Thom S. "Seven Responses to Prevent Pastor Burn Out". 30 de septiembre, 2013, bajo Thom S. Rainer Growing Healthy Churches. Together, http://thomrainer.com/2013/09/seven-responses-to-prevent-pastor-sindrome de burnout/ (consultado: 27 de março de 2017). . Thom S. The Book of Church Growth: History, Theology, and Principles. Nashville, TN: Broadman Press, 1993. , Thom S. "When Pastor Experience Depression". (july 18, 2011). http://thomrainer.com/2011/07/when_pastors_experience_depression/ (consultado: 23 de abril de 2017). . "The Lifecycle of Pastors", en: Sitio oficial de Thom Rainer. november 2011. http://blogs.lifeway.com/cgi-bin/mt/mtsearch.cgi? search=pastor+tenure $\&$ IncludeBlogs $=31 \&$ limit $=20$. (consultado: 14 de junio, 2012).

Robbins, Paul D. When it's Time to Move: A Guide to Changing Churches. The Leadership Lybrary 4. Waco, TX: Word Books, 1985.

Robbins, Stephen. Administración. México: Pearson, 2005. citado en: Silva, Felipe Reynaldo Esteban. "Cultura organizacional, compromisso cristiano y la satisfacción laboral en los docentes de la Universidad Peruana Unión de la ciudad de Lima, Peru, 2013”. Tesis doctoral, Universidad Peruana Unión, 2013.

Robertson, I. T., Cooper, C. L., \& Willians, J. "The Validity of the Occupational Stress Indicator". Work and Stress 4, no. 1, (1990): 29-39.

Rode, Daniel y Isabel Rode. Crescimento: chaves para revolucionar sua igreja. Engenheiro Coelho: UNASPRESS, 2007. . "Los siete signos vitales de crecimiento de Wagner en seis iglesias adventistas hispanas dl sur de California". Tesis doctoral, Fuller Theological Seminary, 1994. 
Rodríguez, Katia Caballero. "El concepto de satisfacción en el trabajo y su proyección en la enseñanza". Revista de currículum y formación del profesorado 6, no 1-2 (2002): 1-10. http://www.ugr.es/ recfpro/rev61COL5.pdf. (consultado: 16 de enero, 2017).

Rosa, Cristiane da, y Carlotto, Mary Sandra. "Síndrome de sindrome de burnout e satisfação no trabalho em profissionais de uma instituição hospitalar". Rev. SBPH [online] 8, no. 2, (2005): 1-15. http://pepsic.bvsalud.org/scielo.php?script=sci_arttext\&pid=S151608582005000200002\&lng=pt\&nrm=iso. (consultado: 04 de enero, 2017).

Salazar, Ronald Alfaro, Girón, Sara Leyton, Solano, Antonio Meza, y Torres, Ivonne Saénz "Satisfacción laboral y su relación con algunas variables ocupacionales en tres municipalidades”. Tesis de maestría, Pontificia Universidad Católica del Perú, 2012

Sales, Ranieri, trad., Manual da igreja adventista do sétimo dia. Tatuí, SP: Casa Publicadora Brasileira, 2016.

Salluca, Luiz Salluca, "Relacion entre Niveles de Satisfacción Laboral y Desenpeño Docente en Instituciones Educativas del Cercano Callao”. Tesis de maestria, Universidad San Ignacio de Loyla, 2010.

Sahlin, Monte. "Sharing our Faith with Friends Without Losing Either: What the Fastest Growing Adventist Churches Know”. Washington: Review and Herald, 1990. Sánchez-Sellero, M. C., Sánchez-Sellero, P., González, M. M. C., y Sánchez-Sellero, F. J. "Características Organizacionales de La Satisfacción Laboral en España”. Revista de Administração de Empresas 54, no. 5, 2014: 538. http://dx.doi.org/10.1590/S0034-759020140507. (consultado: 22 de enero, 2016).

Santos, Jobson D. "Vantagens de pastorados longos". Revista Ministério, no. 5, setembro-outubro, 1998: 9-10.

Sarli, Joel. Crescimento de igreja. São Paulo: Instituto Adventista de Ensino, 1981.

Schaller, Lyle E. Assimilating new members. Nashville, TN: Parthenon Press, 1989. “"When Should the Pastor Move?" Christian Ministry, (July 1980): 26. . 21 Bridges to the 21st Century. Nashville, TN: Abingdon Press, 1994.

Scott, Donald M. "Pastors and Providence: Change Ministerial Styles in Nineteenth Century America". Evanston, III: Seabury-Western Theological Seminary, 1975. Citado en Loomis, Gregory Mark "Key Factors of Pastoral Longevity in the 
Evangelical Free Church of America”. Tesis Doctoral, Trinity Evangelical Divinity School, 2006. En Tren Dissertations. Paper 3256.

http://place.asburyseminary.edu/trendissertations/3256. (consultado: 9 de Agosto, 2016).

Scott, Harold D. "Personal Reflections on Pastoral Sindrome de burnout". Tesis doctoral, Fuller Theological Seminary, 1994.

Serns, Dan. "Por onde devo recomeçar?" Revista Ministério. no. 1. (janeiro-fevereiro, 2010): 10-12.

Seymour, Jody. A Time for Healing: Overcoming the Perils of Ministry. Valley Forge: Judson Press, 1995. Citado en: Williams, Ivan Leander. "Pastoral Sindrome de burnout and Renewal in Seventh-Day Adventist Ministry". Professional Project, Claremont School of Theology, 2002.

Shedd, William Greenough Thayer. Pastoral Theology. Titus Books, 2013. Kindle Edition.

Silva, Itaniel. "Desafio compensador". Revista ministério, no. 497, (novembrodezembro 2011): 5-7.

Somers, George Leslie. "The Ministry Marathon: Exploring Longevity in the Single Pastorate". Tesis doctoral, Acadia Divinity College, 2004.

Soria, V. Relaciones Humanas. México: Editorial Limusa SA, 2004. Citado en: Silva, Felipe Reynaldo Esteban. "Cultura organizacional, compromisso cristiano y la satisfacción laboral en los docentes de la Universidad Peruana Unión de la ciudad de Lima, Perú, 2013”. Tesis doctoral, Universidad Peruana Unión, 2013.

Spector, Paul E. Job Satisfaction: Application, Assessment, Causes, and Consequences. Thousand Oasks, CA: SAGE Publications, 1997. Kindle edition.

Tamayo, A., "Valores organizacionais: sua relação com satisfação no trabalho, cidadania e comprometimento afetivo", Revista de Administração RAUSP 33, no. 3, 1998: 56-63. Citado en Vieira, Valter Afonso, Appio, Jucelia, y Slongo, Luiz Antonio, "Uma análise dos antecedentes da satisfação no trabalho em uma empresa madeireira", Revista de Gestão USP 14, no. 3, (2007): 2.

Taylor, Glenn C. Pastors in Transition: Navigating The Turbulence of Change. Winnipeg: Word Alive Press, 2013.

Torres, Tania. Agridoce: a vida da esposa de pastor. Belo Horizonte: edição do autor, 2009.

Tripp, Paull. "Depression and the Ministry, Part 1: The Setup". The Gospel Coatlition 
(July 11, 2011). https://www.thegospelcoalition.org/article/depression-and-theministry-part-1-the-setup/ (consultado: 23 de abril de 2017).

Turner, R. Edward. "Pastoral Care as a Factor of Church Growth". Citado en Firth, Robert E. (ed.) "Servants for Christ” Berrien Springs: Andrews University Press, 1980.

Vaughan, John N. The Word's Twenty Largest Churches: Church Growth Principles in Action. Grand Rapids, Michigan: Baker Book House, 1984.

Venden, Morris L. Como Jesus tratava as pessoas. Tatuí, SP: Casa Publicadora Brasileira, 1990.

Versteeg, Norman. "Doze anos numa igreja". Revista ministério, no. 1, (janeirofevereiro 1982): 7-8.

Vitello, Paul. "Taking a Break from the Lord's Work". The New York Times, $(1 \mathrm{de}$ Agosto, 2010). http://www.nytimes.com/2010/08/02/nyregion/02sindrome de burnout.html (consultado: 23 de abril de 2017).

Wagner, C. Peter. Descubra seus dons espirituais. São Paulo: Abba Press Editora, 2009.

Wagner, Philip. "The Secret Pain of Pastors". (29 de agosto, 2014).

http://churchleaders.com/pastors/pastor-articles/167379-philip-wagner-secretpain-of-pastors.html (consultado: 24 de abril de 2017).

Warren, Rick, The Purpose Driven Church Without Compromising Your Message \& Mission. Grand Rapids: Zondervan Press, 1995.

Weiss, H., \& Cropanzano, R., “Affective Events Theory: a Theoretical Discussion of the Structure, Causes and Consequences of Affective Experiences at Work", Research in Organizational Behavior 18, 2006: 1-74. Citado en Ronald A. Salazar, Sara L. Gyrón, Antonio M. Solano, Ivonne S. Torrez, "Satisfacción laboral y su relación con algunas variables ocupacionales en tres municipalidades”. Tesis doctoral, Pontificia Universidad Católica del Perú, 2012.

Williamson, Natalie. “A Quantitative Research Study Analyzing Responses or Reactions of Personal and Professional Stress-Related Factors That Can Lead to Ministry Stress or Sindrome de burnout in Chinese Pastors". Tesis doctoral, The University of the Rockies, 2011.

Wiersbe, Warren W. Comentário bíblico expositivo. 6 Vols. Traducido por Susana E.

Klassen. Santo André, SP: Geográfica, 2006.

White, Ellen. Conselhos sobre saúde. Tatuí, SP: Casa Publicadora Brasileira, 1998. 
. Evangelismo. Tatuí: Casa Publicadora Brasileira, 1997.

. Medicina e Salvação. Tatuí, SP: Casa Publicadora Brasileira, 1991.

. Mente, caráter e personalidade. Vol. 2. Tatuí, SP: Casa Publicadora Brasileira, 1989.

. Ministério Pastoral: conselhos aos pastores adventistas. Tatuí, SP: Casa

Publicadora Brasileira, 2015

. Obreiros evangélicos: instruções para todos os que são cooperadores de Deus. Tatuí: Casa Publicadora Brasileira, 1993.

. O desejado de todas as nações. Tautí, SP: Casa Publicadora Brasileira, 1996.

. Parábolas de Jesus. Santo André, SP: Casa Publicadora Brasileira, 1979.

. Patriarcas e profetas. Tatuí, SP: Casa Publicadora Brasileira, 1989.

. Profetas e reis. Tatuí, SP: Casa Publicadora Brasileira, 1996.

. Vida de Jesus. Tatuí, SP: Casa Publicadora Brasileira, 2012.

Williams, Ivan Leander. "Pastoral Sindrome de burnout and Renewal in Seventh-Day Adventist Ministry". Professional Project, Claremont School of Theology, 2002.

Williamson, G. B. Pastores del rebaño: un tratado moderno sobre teología pastoral. Kansas City, MO: Casa Nazarena de Publicaciones, 1992.

Wohlrabe, Lawrence R. “Clergy Sindrome de burnout: Definition, Causes, Incidence, Responses”. Term Paper, Luther Seminary, 2001. Citado en: Ferreira da Silva, Jetro. "O sindrome de burnout pastoral na perspectiva da teologia prática: definições, causas e prevenção". Tesis doctoral, Pontifícia Faculdade de Teologia Nossa Senhora da Assunção, 2006.

Zambelli, Ricardo N. "Implicaciones de la duración de los pastorados en Argentina". Tesis doctoral, Universidad Adventista del Plata, 1997. 